\title{
Novel Wideband 3D Transitions on Liquid Crystal Polymer for Millimeter-Wave Applications up to $100 \mathrm{GHz}$
}

\author{
Amin Rida ${ }^{1}$, Alexandros Margomenos $^{2}$, Terence $\mathrm{Wu}^{1}$, and Manos M. Tentzeris ${ }^{1}$ \\ ${ }^{1}$ GEDC/School fo ECE, Georgia Institute of Technology, Atlanta, GA, 30332-250, USA \\ ${ }^{2}$ Toyota Research Institute North America, Ann Arbor, MI, 48105 ,USA \\ arida@ece.gatech.edu
}

\begin{abstract}
This paper reports on two novel broadband vertical transitions on flexible organic Liquid Crystal Polymer (LCP) substrate which show superior characteristics- Return Loss below $-10 \mathrm{~dB}$, Insertion Loss better than $-2.5 \mathrm{~dB}$ from 60 to $100 \mathrm{GHz}$. The presented Coplanar Waveguide-Coplanar WaveguideMicrostrip (CPW-CPW-MSTRIP) and CPW-CPW-CPW 3D transitions require strategically placed vias and tapering of the CPW ground planes in order to suppress radiation loss and optimize the performance over a very broad frequency range. These transitions are simple to realize and are compatible with low-cost substrate fabrication guidelines allowing for the easy feeding of embedded IC's in 3D modules, especially in compact automotive radar applications and beam-steering wideband antenna arrays. To estimate the effect of practical fabrication variations, a sensitivity analysis was performed against the lateral misalignment of via to pad defined by commercial fabrication houses and shows a worst case scenario of a $5 \mathrm{~dB}$ RL and $0.2 \mathrm{~dB}$ IL degradation compared to the ideal case. The measurement results of the reported transitions exhibit very good performance up to $100 \mathrm{GHz}$ and show reasonable agreement with simulation.
\end{abstract}

Index Terms - coplanar waveguide, microstrip, sensitivity analysis, transition, via, sensitivity, millimeter-wave, 3D interconnects.

\section{INTRODUCTION}

$\mathrm{T}$ HE demand for low-cost, wideband, and miniaturized RF 3D transitions that are compatible with current stateof-the-art design rules on flexible material calls for superior performance in mm-wave frequencies, especially as the operating frequencies are steadily growing in order to achieve increasingly higher bandwidth [1]. 3D transitions are very important, especially in highly integrated 3D RF front-ends, similar to Fig.1, that require layer-to-layer interconnects between embedded IC's (e.g. transceiver, DSP chips) and integrated passives, such as antennas and filters. Realizing compact interconnects with low insertion and reflection loss over an ever increasing frequency range, sometimes reaching above $100 \mathrm{GHz}$, is paramount for high-sensitivity RF applications. Such vertical architectures could potentially eliminate bulky highly-loss waveguide transitions that are currently used in many broadband millimeter-wave systems, especially in the field of automotive radars [2].

The purpose of this paper is to describe a method for creating easy-to-fabricate low-cost 3D transitions on the flexible organic Liquid Crystal Polymer (LCP) [1] for use with mmwave (up to $100 \mathrm{GHz}$ ) front-end $[3,4]$ applications such as broadband high-speed wireless LAN[5], automotive radar and imaging systems. This material has recently gained much consideration as a potential high-performance microwave medium with excellent "dual" (substrate and packaging) functionality due to its excellent electrical, mechanical and hermetic properties. For example, the frequency stability of its low dielectric constant, as well as its low loss tangent up to $110 \mathrm{GHz}$ are very attractive for developing mm-wave circuitry [1]. In addition, LCP can be easily laminated and micromachined to form 3D multi-layer modules. Finally, its coefficient of thermal expansion (CTE) can be adjusted through thermal treatment to offer a low-stress packaging alternative. Due to its near-hermetic characteristic, LCP can be easily used for the integration of embedded bare IC's without the use of additional packaging material, something that necessitates the development of broadband low-loss 3D CPWto-CPW and CPW-to-MStrip interconnects.

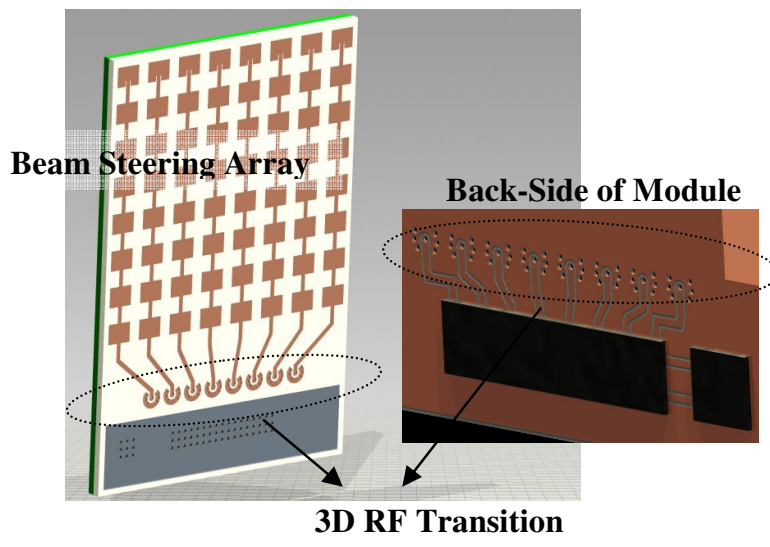

Fig. 1. Wideband Vertical CPW-CPW-MSTRIP Transition (left) top view (right) bottom view 
The proposed design achieves a very good wideband RF performance by utilizing a tapered ground plane and by placing grounding vias in appropriate locations in order to suppress parasitic modes and eliminate radiation losses due to open-end effects. Furthermore, the via pads and gaps are optimized so as to match the series inductance of the via transition and maintain a $50 \mathrm{Ohms}$ characteristic impedance throughout the transition. This allows us to achieve a very broadband response up to $100 \mathrm{GHz}$, which is much better than the conventional waveguide transitions used in some mm-wave systems and which are significantly larger in dimensions [6,7]. The proposed designs take into consideration the design rules and tolerances that are found in commercially available LCP fabrication techniques and are the first to demonstrate a 3D transition on flexible substrate up to $100 \mathrm{GHz}$.

\section{WIDEBAND 3D TRANSITIONS}

Two wideband 3D transitions, one for Coplanar Waveguide (CPW)-CPW-MSTRIP Line and another for CPW-CPW-CPW Line will be presented on LCP. Both of these consist of a vertical and a horizontal transition. All dimensions on the

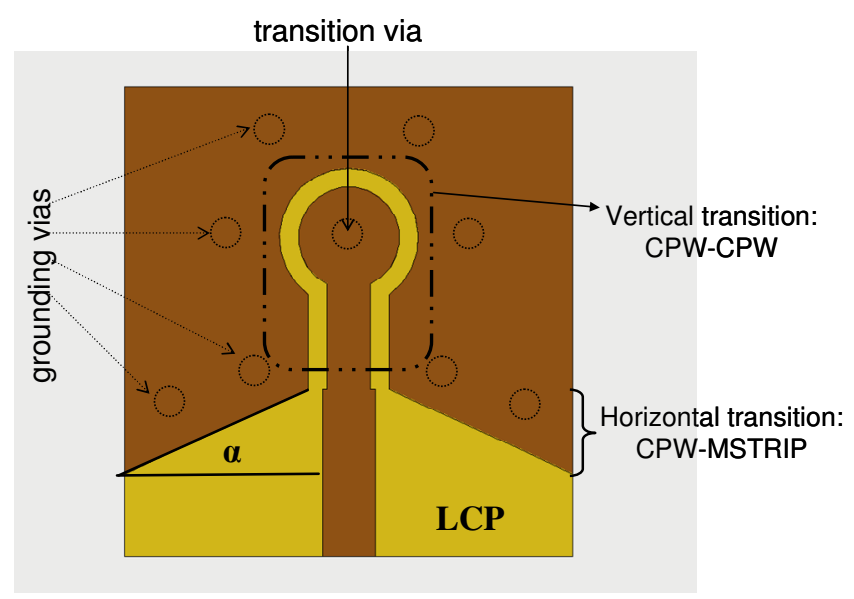

(a)

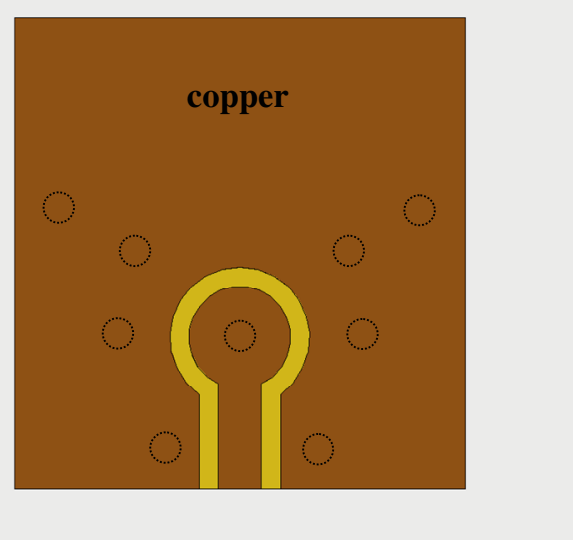

(b)

Fig. 2. Wideband Vertical CPW-CPW-MSTRIP Transition (a) top view (b) bottom view

designed structures are based on existing commercially available design rules and the use of mechanical drilling for via formation.

\section{A. CPW-CPW-MICROSTRIP Transition}

The design consists of one via-based vertical transition from center to center conductors, that is used to connect the transmission lines printed on both sides of a single LCP layer ( $\varepsilon$ r of 3.16 and thickness of 4 mils), as well as grounding vias connecting the top and bottom ground layers. Shown in Fig.2 is the schematic of the design with the specific via placements. The bottom layer (Fig. 2b) starts as a CPW launch with characteristic impedance $\mathrm{Z}_{0}$ of $50 \mathrm{Ohms}$, realized appropriately choosing the signal line width and the gap between the signal line and the ground. The CPW slot width ( $~ 85 \mathrm{um})$ is responsible for maintaining a desired CPW mode for the wide frequency throughout the CPW section of the transmission line (top and bottom), so it is kept at a constant value around the via pad, resulting in minimum field reflections and very good impedance matching.

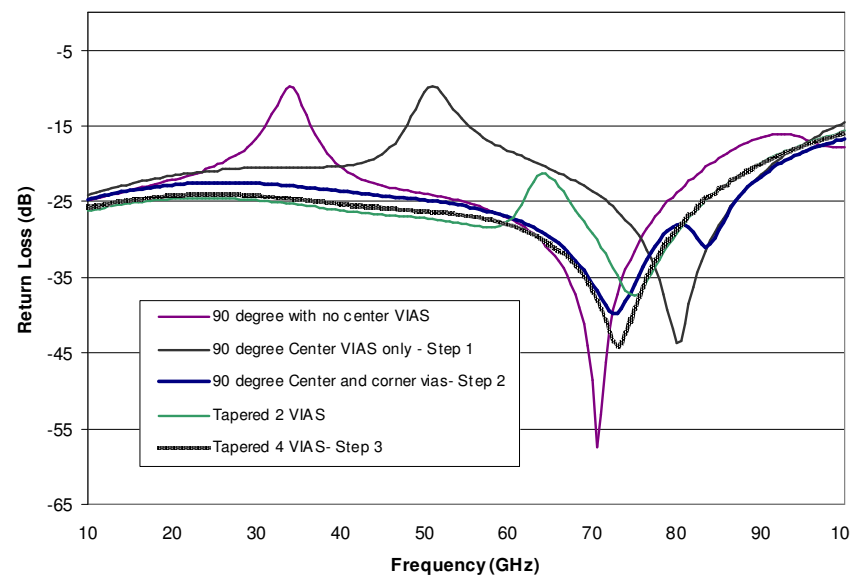

(a)

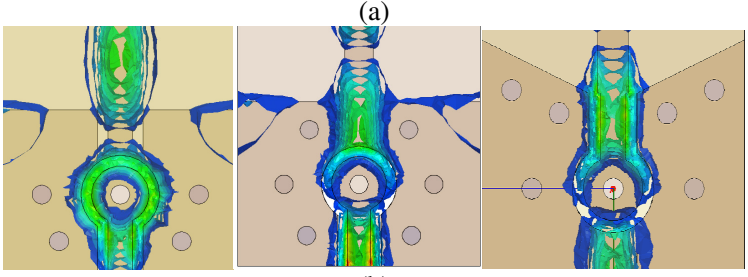

(b)

Fig. 3. Transition Synthesis steps (a) S-parameters: Return Loss and Insertion Loss of 3D Transition (b) E Field Distribution for design steps 1, 2, and 3 as shown in (a)

The transition via lies within a wide circular disk shape to accommodate for the via pad, which is required by the fabrication process (6 mils radius plus the via radius) for the via metallization. The specific dimensions of the transition via as well as the via pad that surrounds it were chosen in accordance to the RF design requirements as well as the fabrication design guidelines and tolerances.

The CPW ground planes on the top layer have been tapered at an angle $\alpha$ of $\sim 27$ degrees with a ground width of $4.25 \mathrm{x}$ the microstrip signal line width as shown in Fig. 2a. and vias with a radius of 3 mils have been strategically placed around the transition and the ground plane edges in order to achieve the optimum broadband RF performance. This is achieved by minimizing the return loss (RL) and insertion loss (IL) due to impedance mismatches and by eliminating the radiation loss 
due to the open-end effects. A step by step process shown in Fig. 3 was used in order to achieve the optimal results for the transition. First the shape and dimensions of the via, via pad, and slot width were chosen in order to achieve minimum return loss for a wideband operation. Then, the number of used vias was minimized in order to reduce the fabrication cost and still remain consistent with the design rules. This is illustrated in Fig. 3, where both grounding vias and tapering of the CPW gound plane had to be implemented. Furthermore, grounding vias close to the edge of the $\mathrm{CPW}$ ground planes were used in order to suppress radiation due to open end effects.

\section{B. $C P W-C P W-C P W$ Transition}

The same design approach, with the addition of vias for ground equalization between the top and bottom layers, was followed for designing a wideband CPW-CPW-CPW transition. The simulated return loss is below $-15 \mathrm{~dB}$ for the 60-90 GHz frequency range (Fig. 4). For this design the major focus was the selection of the optimum number of and spacing between the vias in the CPW section in order to suppress the parallel plate mode. Maintaining a $600 \mu \mathrm{m}$ spacing with a via radius of 3 mils was sufficient in order to both achieve good performance and maintain a low cost fabrication process.

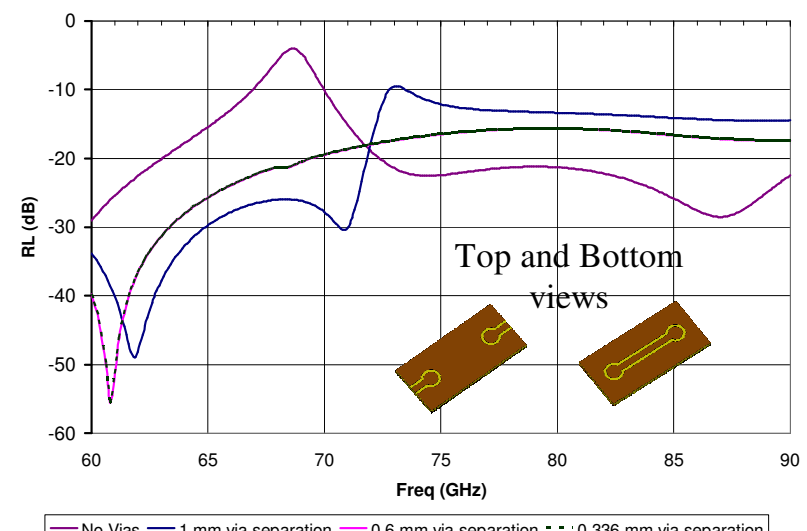

- No Vias $-1 \mathrm{~mm}$ via separation $-0.6 \mathrm{~mm}$ via separation $\cdots \cdot 0.336 \mathrm{~mm}$ via separation

Fig. 4. S-parameters: Return Loss and Insertion Loss of 3D Transition

\section{MEASURED RESULTS}

The photographs of the fabricated circuits are shown in Fig. 5 where both the CPW-CPW-MSTRIP and the CPW-CPW transitions are included. The measurements were performed on a Agilent 8510C Vector Network Analyzer that in turn uses the Agilent 8510XF millimeter-wave controller and Agilent E7352R $110 \mathrm{GHz}$ test head or millimeter-wave mixing modules. In order to be able to use $150 \mu \mathrm{m}$-pitch probes a narrower CPW RF launch was incorporated in the designs. The effect of these RF launches was not de-embedded and it is included in all the measurements.

The measured and simulated S-parameters for the CPW-CPWMSTRIP transition are plotted in Fig. 6 demonstrating a wideband operation from 60 to $100 \mathrm{GHz}$ shown by the $-10 \mathrm{~dB}$ bandwidth. It has to be noted that both $\mathrm{RL}$ and IL were affected considerably due to fabrication and measurement setup issues. In the fabricated structures, it was observed that metal over-etching has occurred for both CPW and microstrip metal lines; also, the vertical vias have been misplaced off- center within the pad; both issues are studied in further detail in the sensitivity analysis section of this paper, hence explaining the experimental shift of the minimum points on the RL plot. The over-etching is very critical for mm-wave frequency, especially in the CPW RF launches as well as in the width of the MicroStrip line, so it is expected to be the main factor of the difference in RL and IL levels. Another issue affecting significantly the measurements was that in order to minimize the effect of the metal chalk, the measurement setup, shown in Fig. 5, used an LCP layer of 16 mils thickness surrounding the fabricated structures, which in turn was placed

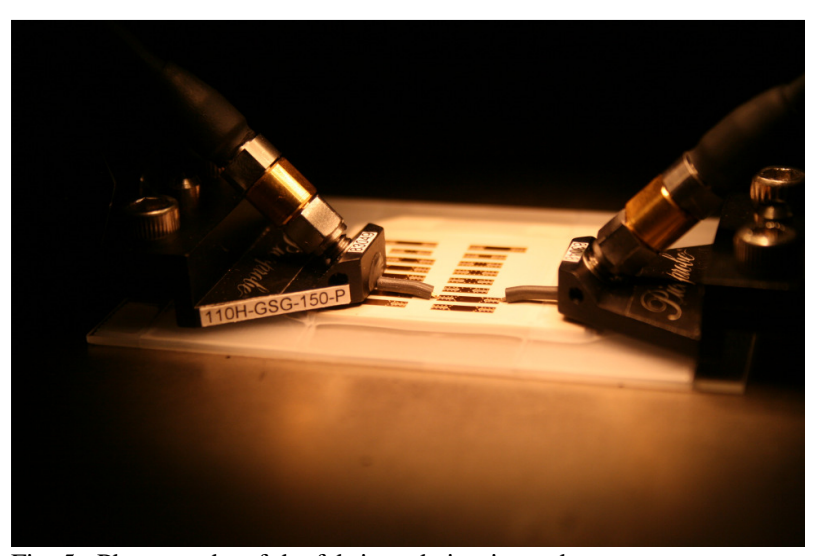

Fig. 5. Photographs of the fabricated circuits under measurement

on a glass platform. This multidielectric configuration changed the effective dielectric constant of the transition substrate realizing a "superstrate" for the transmission lines in the lower side. Thus, it is another significant factor responsible for the difference in periodicity in RL plot as shown in Fig. 5. The measured insertion loss is $-3.7 \mathrm{~dB}$ at $76.5 \mathrm{GHz}$ for the back-to-back transition which translates to $-1.6 \mathrm{~dB}$ for a single transition as illustrated in Fig.2. the simulated E-field from HFSS is presented demonstrating that all the fields are confined along the signal line, with minimal radiation due to the open-end effects.

Fig. 6 presents the measured and simulated response of the CPW-CPW-CPW transition. A -10 dB S11 bandwidth covers the whole frequency range from $60-100 \mathrm{GHz}$ and the measured insertion loss is $-2.9 \mathrm{~dB}$ at $76.5 \mathrm{GHz}$ for the back-to-back transition which translates to $-1.3 \mathrm{~dB}$ for a single transition.

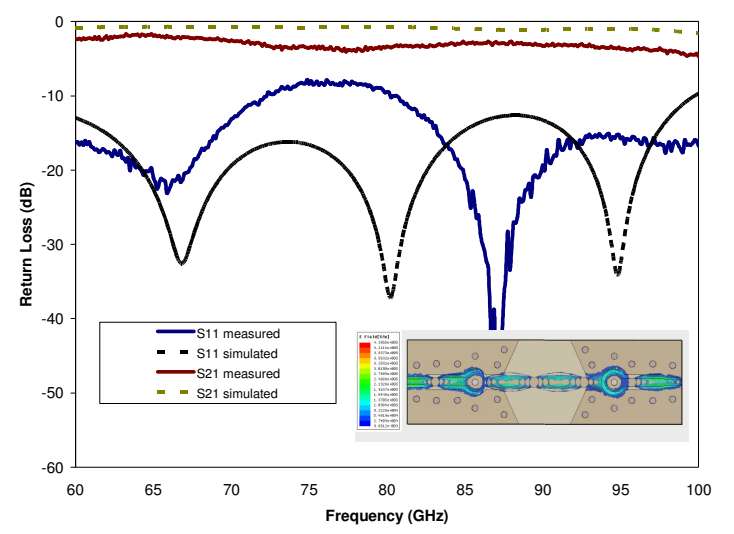

Figure 6. $\mathrm{S}_{11}$ and $\mathrm{S}_{21}$ for CPW-CPW-MSTRIP Back-to-Back Transition 


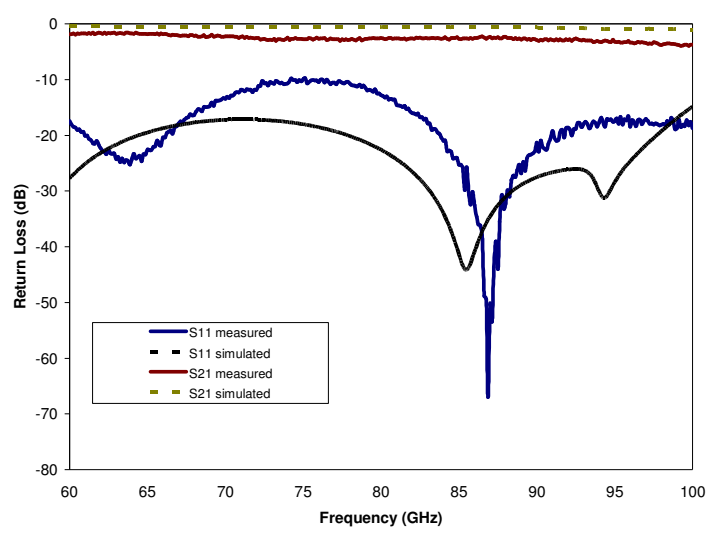

Figure 7. $S_{11}$ and $S_{21}$ for CPW-CPW-CPW Back-to-Back Transition

\section{Sensitivity ANALYSIS}

In order to examine the viability of the presented design in a large scale production environment, a sensitivity analysis based on the tolerances and design rules provided by the manufacturer was performed. Figure 7 summarize the results for return and insertion loss respectively. It can be seen that even in the worst via-to-pad misalignment case of \pm 3 mils the CPW-CPW-MSTRIP transition maintains an acceptable performance at $76.5 \mathrm{GHz}$ (simulated Return loss below $-15 \mathrm{~dB}$ and insertion loss of $-0.35 \mathrm{~dB}$ ), despite the fact it could cause a shift in the central design frequency. The authors plan to present at the conference detailed results about different radius of flexing curvature for both introduced interconnects.

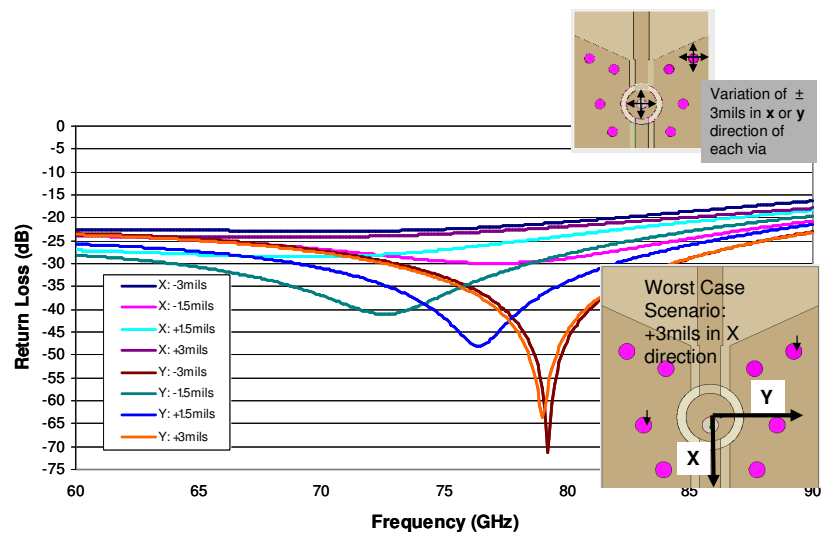

(a)

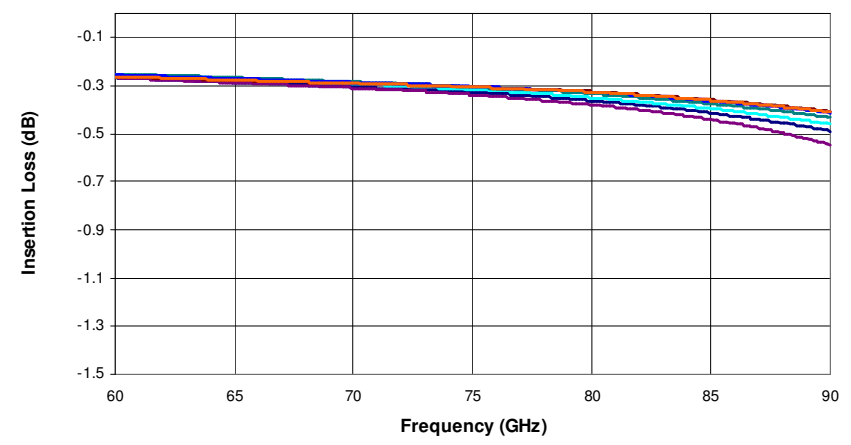

(b)

Fig. 8.: Return Loss and Insertion Loss of 3D Transition for off-center vias

\section{CONCLUSIONS}

This paper presented two novel broadband 3D vertical transitions on flexible organic Liquid Crystal Polymer substrate that demonstrate an excellent performance at X/W-band (Return Loss below -10 dB, Insertion Loss better than $-2.5 \mathrm{~dB}$ from 60 to $100 \mathrm{GHz}$ ). The transitions are implemented on a single LCP layer, are easily realizable, and are compatible with low-cost substrate fabrication guidelines. This makes them very appealing for cost sensitive applications. The presented design is a critical component of any highly integrated 3D RF front-end, that demonstrates the capabilities of LCP to form high performance, low-cost, System-in-Package conformal broadband architectures up to $100 \mathrm{GHz}$.

\section{ACKNOWLEDGMENT}

The fabrication of the circuits was done at Dynaco Corporation. The authors would like to thank Gary Bachler of Dynaco for his assistance with the fabrication.

\section{REFERENCES}

[1] D. C. Thompson, O. Tantot, H. Jallageas, G. E. Ponchak, M. M. Tentzeris, and J. Papapolymerou, "Characterization of liquid crystal polymer (LCP) material and transmission lines on LCP substrates from 30-110 GHz," IEEE Trans. Microwave Theory Tech., vol. 52, no.4, pp. 1343-1352, April 2004.

[2] M. Steinhauer, H-O Ruov, H. Irion, and W. Menzel, "Millimeter-Wave Radar Sensor Based on a Tranceiver Array for Automotive Applications", IEEE Transactions on Microwave Theory and Techniques, vol. 56, no.2, pp.,xx-xx, February 2008.

[3] A. Margomenos, Y. Lee, L.P.B. Katehi, "Wideband Si Micromachined Transitions for RF Wafer-Scale Packages", IEEE Topical Meeting on Silicon RF Components SiRF2007, Long Beach, CA, January 2007.

[4] A. Stark, A.F. Jacob, "A Broadband Vertical Transition for MillimeterWave Applications”, European Microwave Conference 2008 Digest, pp. 476-479, October 2008, Amsterdam, NETHERLANDS.

[5] B. Pan, Y. Li, M. M Tentzeris and J. Papapolymerou, "HighPerformance Millimeter-wave Surface Micromachined Cavity Filters for WPAN Applications", IEEE Transaction on Microwave Theory and Technology, Vol.56, No.4, pp.959-970, April 2008

[6] W. Mayer, M. Meilchen, W. Grabherr, P. Nuchter, and R. Guhl, "EightChannel 77-GHz Front-End Module with High Performance Synthesized Signal Generator for FMCW Sensor Applications", IEEE Transactions on Microwave Theory and Techniques, vol. 52, no 3, pp. 993-1000, March 2004.

[7] Y. Deguchi, K. Sakakibara, N. Kikuma, H. Hirayama, Design and optimization of mm-wave microstrip-to-waveguide transition operating over broad frequency bandwidth, IEICE Transactions on Electronics, vol. E90-C, no.1, pp. 157-164, January 2007. 